\title{
TMS stimulation over the inferior parietal cortex disrupts prospective sense of agency
}

\author{
Valérian Chambon • James W. Moore • \\ Patrick Haggard
}

Received: 28 June 2013/Accepted: 11 August 2014/Published online: 19 August 2014

(c) Springer-Verlag Berlin Heidelberg 2014

\begin{abstract}
Sense of agency refers to the feeling of controlling an external event through one's own action. On one influential view, sense of agency is inferred after an action, by "retrospectively" comparing actual effects of actions against their intended effects. However, it has been recently shown that earlier processes, linked to action selection, may also contribute to sense of agency, in advance of the action itself, and independently of action effects. The inferior parietal cortex (IPC) may underpin this "prospective" contribution to agency, by monitoring signals relating to fluency of action selection in dorsolateral prefrontal cortex (DLPFC). Here, we combined transcranial stimulation (TMS) with subliminal priming of action selection to investigate the causal role of these regions in the prospective coding of agency. In a first experiment, we showed that TMS over left IPC at the time of action selection disrupts perceived control over subsequent effects of action. In a second experiment, we exploited the
\end{abstract}

V. Chambon and J. W. Moore contributed equally to this work.

V. Chambon $(\bowtie)$

Laboratoire de Neurosciences Cognitives, Institut Jean Nicod, Ecole Normale Supérieure, CNRS, 29, rue d'Ulm,

75005 Paris, France

e-mail:valerian.chambon@ens.fr

V. Chambon

Department of Neuroscience, Biotech Campus-University

of Geneva, 1211 Geneva, Switzerland

J. W. Moore

Department of Psychology, Goldsmiths College, University of London, London SE14 6NW, UK

P. Haggard

Institute of Cognitive Neuroscience, University College London, London WC1N 3AR, UK temporal specificity of single-pulse TMS to pinpoint the exact timing of IPC contribution to sense of agency. We replicated the reduction in perceived control at the point of action selection, while observing no effect of TMS-induced disruption of IPC at the time of action outcomes.

Keywords Action selection - Agency - Inferior parietal cortex $\cdot$ Dorsolateral prefrontal cortex $\cdot$ Single-pulse TMS

\section{Introduction}

Healthy adults generally feel a sense of control over their own actions, and over the effects of those actions-a feeling that is classically referred as "sense of agency". Theoretical accounts identify several components of the sense of agency, including the feeling that actions are selfcaused, involve one's own body, and depend on one's voluntary choices (Haggard and Tsakiris 2009). Psychometric studies generally consider agency as a synthetic experience in which these different components form a single composite mental content (Haggard and Chambon 2012). Psychometric studies disagree, however, over whether agency is primarily a feeling, a judgement, or a metacognitive experience (Synofzik et al. 2008; Metcalfe and Greene 2007).

One influential computational model has proposed that sense of agency is computed by matching predicted and actual sensory consequences of movement (Blakemore et al. 1998; Farrer et al. 2008; Moore and Haggard 2008; Sato 2009). Thus, the sense of agency is strong when there is a close match between the predicted and the actually experienced consequences of an action, and is reduced when predicted and experienced consequences do not match (Linser and Goschke 2007; David et al. 2008; Farrer 
et al. 2008). According to this view, sense of agency occurs late-after sensory evidence about the consequences of action becomes available. This view has received considerable empirical support from studies showing that temporal (Franck et al. 2001; MacDonald and Paus 2003; Leube et al. 2003; David et al. 2007) and spatial discrepancies (Fourneret and Jeannerod 1998; Farrer et al. 2003; Synofzik et al. 2006; David et al. 2007) between making an action and viewing visual feedback of the action reduce the sense that the observed action is one's own.

More recently it has been suggested that earlier processes, linked to action selection and preparation, can also contribute to sense of agency. In particular, subliminal priming of actions was shown to increase the feeling of control over the effects of those actions (Wenke et al. 2010; Chambon and Haggard 2012; Chambon et al. 2013), even though the primes did not improve the predictability of the effects themselves. Typically, participants are asked to respond to a left- or right-pointing arrow with a left or right keypress, respectively. The keypress causes a colour patch to be presented on a screen after a short delay. Participants are asked to judge how much control they feel over the patch of colour that follows their keypress action. Prior to the directional target a subliminal prime is presented, unbeknownst to the subject. The subliminal prime is a directional cue either congruent or incongruent with the target. Judgements of control over the colour presented after action are modulated by prime-target congruence: when the prime is compatible with the instruction arrow and with the keypress response, participants experienced more control over the effects of action than when the prime was incompatible with the target and the keypress (Wenke et al. 2010). Since the relation between primes and colour effects was statistically identical for compatible and for incompatible primes, this effect cannot be linked to the anticipation of the effects of action, such as the well-known phenomenon of ideomotor priming (Gentsch and SchützBosbach 2011). Instead, the influence of prime compatibility is thought to depend on action selection processes, which necessarily occur prior to movement. Specifically, incompatible prime-target pairings are thought to decrease the fluency of action selection relative to compatible prime-target pairings, which in turn reduces the subsequent sense of agency. Results from action priming experiments thus suggest that the sense of agency is partly prospective, arising at the action selection stage, and not purely due to retrospective matching occurring after the effects of action are known.

A recent fMRI study by Chambon et al. (2013) has highlighted specific brain regions that may underpin this influence of action priming on prospective agency. The results showed a network involving left dorsolateral prefrontal cortex (DLPFC) and left inferior parietal cortex (IPC-more specifically, the angular gyrus). According to the authors, DLPFC activation would reflect willed action (see also Frith et al. 1991). In contrast, DLPFC deactivation would signal dysfluency in the selection of willed action (as a consequence of prime-target incompatibility), resulting in a diminished sense of control over subsequent action outcomes. The IPC would monitor these signals relating to fluency or dysfluency of action selection emanating from DLFPC and use them to (pre)construct an experience of agency. Importantly, under this interpretation, this monitoring of fluency signals by IPC occurs prior to actions and their sensory consequences.

Although informative, this study is limited in two key ways. First, the evidence is indirect, because of the correlational nature of fMRI. Secondly, it is not possible to pinpoint the precise time at which these brain regions are involved owing to the relatively poor temporal resolution of fMRI. Nevertheless, the issue of timing is important for understanding how sense of agency is computed. Studies of implicit agency using chronometric measures have suggested that sense of agency has both a retrospective component, triggered by the occurrence of action effects, and a prospective component, that is present even if the action effect is omitted (Moore and Haggard 2008; Voss et al. 2010). We hypothesised that the contribution of action selection fluency would occur predictively, in advance of the action outcome. Note that predictive and retrospective processes may be distinguished either by their dependence on outcome occurrence-such as in studies where the actual action effect is omitted (Moore and Haggard 2008)—or by their timing. In the present study, the crucial information for prospective agency occurs prior to action, in action selection circuits. Thus, prospective agency does not require the action or the outcome to be registered, yet it can influence agency subsequently experienced by the subject over the outcome (Chambon et al. 2013; Sidarus et al. 2013).

We here addressed these two limitations by combining single-pulse transcranial magnetic stimulation (TMS) with subliminal priming of action selection and judgements of control over action effects. If TMS-induced disruption of IPC and/or DLPFC influences perceived control over action effects, this will provide direct evidence that these regions are causally involved in sense of agency. Furthermore, we can exploit the temporal specificity of singlepulse TMS to pinpoint when these regions are involved, at the time of action selection or at the time of action effects.

We ran two experiments. In the first we compared the effects of TMS over left IPC and left DLFPC on action selection processing, by linking TMS to the presentation of the arrow target. We made directional predictions based on previous fMRI results. 
First, we found that DLPFC activity was associated with signalling dysfluency of action selection as a consequence of prime-target incompatibility, resulting in a relative deactivation of DLPFC on incompatible trials (Chambon et al. 2013). Therefore, impairing the DLPFC-mediated signalling of prime-target conflict should alter the reduction in agency on incompatible, relative to compatible, trials.

Secondly, because IPC activation correlated with sense of control only on incompatible trials, we assumed that this area monitored signals of action selection conflict generated by DLPFC (Chambon et al. 2013). In this case, disruption of IPC should prevent this region from monitoring any conflict-related signals from DLPFC, and hence reduce the tendency for incompatibility primes to influence judgements of control.

We found that although stimulation had an effect in the predicted direction for both brain regions, it was only significant for IPC. In "Experiment 2" we therefore focussed on IPC and explored when it contributed to judgement of control. We compared the effects of TMS over left IPC at the time of action selection (directional arrow presentation), action execution (keypress) and action effect (colour presentation). To keep the timing of the TMS pulse constant across prospective (response and target onsets) and retrospective (effect onset) conditions, stimulation was delivered $70 \mathrm{~ms}$ similarly after arrow presentation, keypress, and colour presentation. Note that the duration of the resulting disruption to IPC processing is not fully known, but visual studies suggest disruptive effects of TMS last for around $100 \mathrm{~ms}$ (Thut et al. 2003), and up to $200 \mathrm{~ms}$ when single TMS pulse is applied over motor sites (Sanger et al. 2001), while TMS earlier than $60 \mathrm{~ms}$ would be ineffective (Amassian et al. 1998). We therefore reasoned that stimulating the IPC $70 \mathrm{~ms}$ after stimulus or response onset should have hindered any processing in this region up to 170-270 ms after that onset. This duration is consistent with findings of a self-other distinction based on corollary discharge occurring $100 \mathrm{~ms}$ post-stimulus in primary areas (e.g., Martikainen et al. 2005), or with the classical and wellestablished index of early matching processes (mismatch negativity) occurring with a typical latency of 150-250 ms post-stimulus (Garrido et al. 2009). In this second experiment, we replicated the reduction in compatibility effects for IPC stimulation at the point of action selection shown previously in "Experiment 1". This reduction was also present for stimulation at the point of action execution. Importantly, however, the compatibility effect was not reduced by IPC stimulation at the time of action effect presentation.

\section{Experiment 1}

Material and methods

\section{Participants}

Twelve right-handed participants ( 7 females and 5 males aged 21-42 years), with normal or corrected-to-normal vision, were recruited to participate in the study. They provided written informed consent prior to the experiment and were paid $£ 10$ for their participation. All participants were without any known contra-indications to TMS (Keel et al. 2001). The experiment was approved by the UCL Research Ethics Committee and has therefore been performed in accordance with the ethical standards laid down in the 1964 Declaration of Helsinki.

\section{Apparatus and materials}

The visual display was presented on a $19^{\prime}$ computer screen (display mode $=800 \times 600 \times 32,60 \mathrm{~Hz})$. The experiment was programmed and stimulations were delivered using the software Presentation (Neurobehavioral Systems, Albany, California, http://www.neurobs.com). Primes consisted of grey left- or right-pointing arrows that were followed and superimposed by metacontrast masks of the same luminance. The metacontrast masks also consisted of arrows that pointed to the left or the right (see Fig. 1). Participants responded to the masks with keypress actions on the computer keyboard, using their left or right index finger. Prime and mask stimuli could appear randomly above or below fixation to enhance the masking effect (Vorberg et al. 2003). Effects were circular colour patches of red, green, blue, or yellow. All stimuli appeared on a grey background.

Examples of each (left and right) mask stimulus were presented during experimental instruction so that participants would become acquainted with the target stimuli. No reference was made to the existence or appearance of the primes.

\section{Behavioural task}

The paradigm was based on that used previously by Chambon et al. (2013). The participants' task was to judge, by pressing the left or right keys, how much control they had over colour-effect stimuli that followed their keypress actions. The metacontrast masks that served as targets consisted of arrows that unambiguously pointed to the left or to the right. Subliminal primes also consisted of left- or rightpointing arrows. Participants were required to press the key that corresponded to the direction of the mask/target (Fig. 1). 
Fig. 1 a, b Experimental procedure. a Sites of TMS stimulation (based on coordinate locations from Chambon et al. 2013).

b Example trials. Upper panel prime and action are compatible. Lower panel prime and action are incompatible. Prime and masks could appear randomly above or below fixation on each trial. The appearance of the effect was randomly jittered 100,300 , or $500 \mathrm{~ms}$ after the keypress action to increase the range of judgements of perceived control. TMS was delivered $70 \mathrm{~ms}$ after mask/target onset

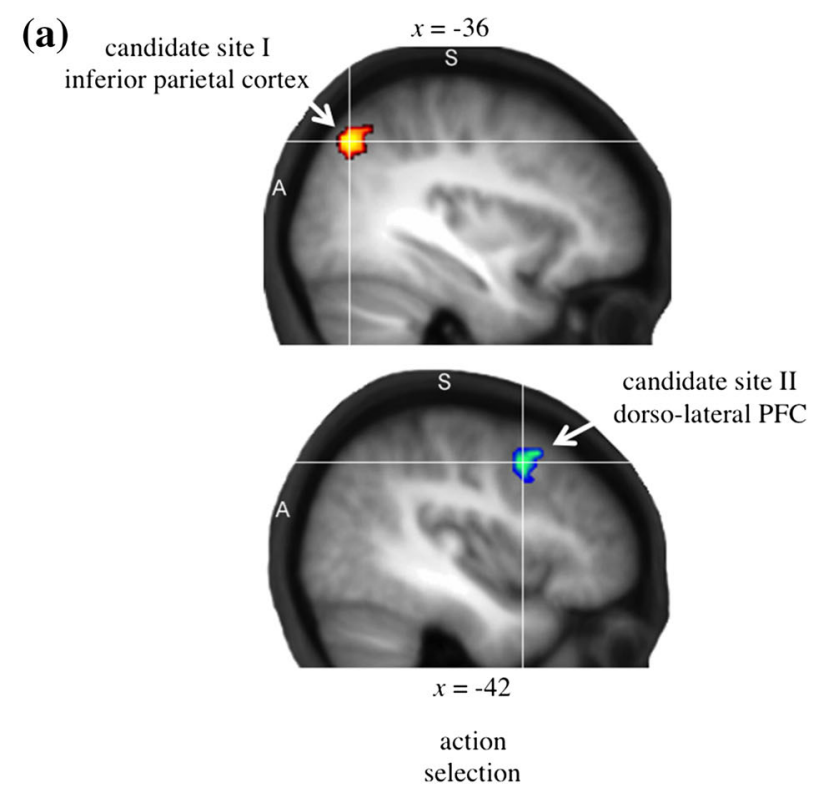

(b)
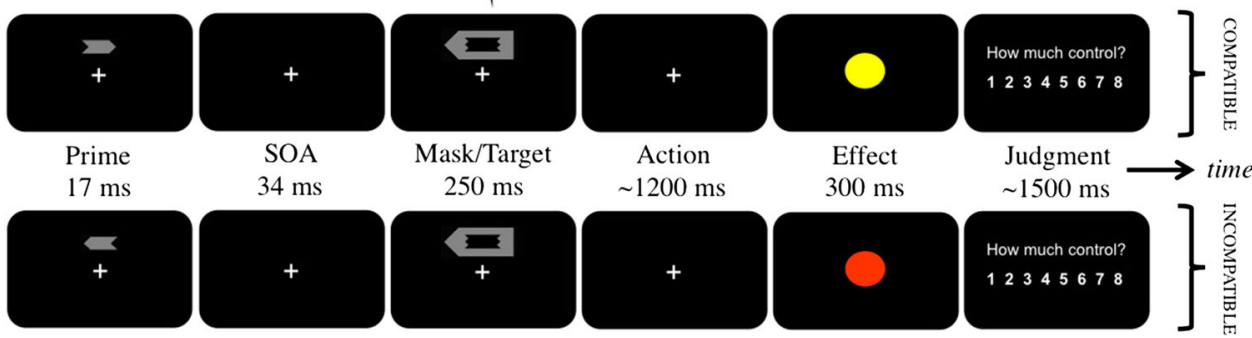

Action $\sim 1200 \mathrm{~ms}$

Effect Judgment $\longrightarrow$ time $+$ $\sim 1500 \mathrm{~ms}$
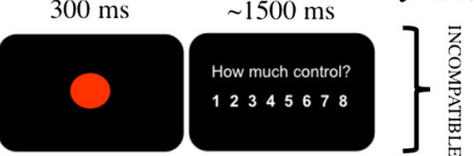

On half of the trials in each block at random, the prime and the mask/target (and therefore also the manual response, assuming that participants responded correctly) were compatible, while on the remaining trials they were incompatible. On prime-response compatible trials, the direction of the prime corresponded to the direction of the mask/target, and hence signalled the same response. On incompatible trials, prime and mask/target pointed in different directions.

Action effects consisted of coloured circles that appeared on the screen 100,300 or $500 \mathrm{~ms}$ after the response. This jitter was introduced because action-effect delays strongly influence sense of agency (Haggard et al. 2002; Wenke et al. 2010), and was therefore expected to induce variations in the sense of control and increase the range of participants' control ratings. The distribution of jitter was the same for all conditions, and thus orthogonal to the manipulation of prime-response compatibility.

Coloured circles were of four different colours (red, green, blue, and yellow). The colour that participants saw on each trial depended whether the trial was prime-target compatible or prime-target incompatible. In each block, two colours (one for each hand) were assigned to prime- compatible responses, another two colours to primeincompatible responses. Compatible effect-colours consistently followed compatible prime-target combinations (e.g., the colour red was shown when a left mask/target followed a left-pointing arrow prime). Incompatible effectcolours were consistently mapped to targets that did not correspond to the direction of the prime (e.g., the colour yellow was shown when a left mask/target followed a right-pointing arrow prime). Colours were rotated through compatibility conditions via a Latin square such that, across all six blocks, each colour appeared in each compatibility condition for each hand. After the colour effect was displayed, at the end of each trial, participants judged how much control they felt they had over the colour effect using a scale ranging from 1 (no control) to 8 (complete control).

When making their judgment, participants were explicitly given the instruction to judge the extent to which they thought they had controlled the appearance of the coloured patch through their action. The control judgement therefore concerned the causal relationship between the action and the consequent effect, rather than simply the effect, or simply the action alone. Such control judgements are a 
standard way of assessing retrospective agency (Synofzik et al. 2008).

\section{Timeline}

Each trial began with a central fixation cross which remained visible until the colour-effect stimulus appeared. Primes were presented for $17 \mathrm{~ms}$, followed by the mask after an SOA (Stimulus Onset Asynchrony) of $34 \mathrm{~ms}$. These parameters were chosen because extensive previous studies consistently showed that conscious perception of prime direction is impossible with these temporal exposures (e.g., Vorberg et al. 2003; Lingnau and Vorberg 2005) and with exactly the same stimuli (Chambon et al. 2013; Chambon and Haggard 2013; Sidarus et al. 2013). In particular, the prime-mask asynchrony strongly influences prime visibility. Vorberg et al. (2003) found that participants could not report the identity of the primes at primemask SOAs from 14 to $70 \mathrm{~ms}$, even after extended practice of more than 3,000 trials. Moreover, none of our participants reported seeing anything prior to the target in a postexperiment debriefing, even when the prime was explicitly mentioned.

Mask/target duration was $250 \mathrm{~ms}$. Participants were asked to respond as fast as possible to the target arrows. The response window was set to $1,200 \mathrm{~ms}$. If participants failed to respond within this time window, or made an incorrect response to the mask/target, they saw a black $\mathrm{X}$ instead of a coloured circle. The coloured patches showing action effects remained on the screen for $300 \mathrm{~ms}$. After a jittered delay (grey background), a rating scale appeared for 1,500 ms, allowing the participant to judge the level of control she felt over the colour patch. Again, if participants failed to make their rating within this time window, they saw a black $X$. Once the participant made her control judgment, the rating scale was replaced by a fixation cross until the end of the $1,500 \mathrm{~ms}$ response window. The fixation cross persisted for a 3,000 ms inter-trial interval. Each block ended with a pause lasting $30 \mathrm{~s}$.

TMS was applied $70 \mathrm{~ms}$ after the onset of the mask/ target to DLPFC, IPC or as Sham stimulation. The duration of the resulting disruption to IPC processing is not fully known, but visual studies suggest disruptive effects of TMS last for around $100 \mathrm{~ms}$ (Thut et al. 2003). The order of the location of stimulation (DLPFC, IPC, or Sham) was counterbalanced across subjects, and is described in the next section. The experiment consisted of 6 blocks of 48 trials each. When an error occurred in a trial, the corresponding trial was repeated at the end of each block (up to 5 error trials per block). Repeating error trials ensured that all colours were seen equally often, even if participants made response errors.

\section{TMS methods}

(a) Localisation of brain sites Single-pulse TMS was delivered using a MagStim 200 magnetic stimulator and a 70-mm figure-of-8 coil (MagStim, Whitland, UK). We aimed to stimulate left IPC and left DLPFC, by placing the coil above activation peaks found in a previous fMRI study using the same behavioural task (Chambon et al. 2013). These peaks did not include the anterior cingulate cortex (ACC), although prime-target incompatibility in the task clearly involved conflict signalling and monitoring. However, this absence of ACC activation is consistent with studies showing that only conscious, but not subliminal, conflict, recruits the ACC (e.g., Dehaene et al. 2003).

The activation peak for left IPC $(x, y, z=-36,-69,45$ in MNI space) lay in the inferior parietal lobule (Brodmann area 39), near the superior edge of the temporal lobe, and immediately posterior to the supramarginal gyrus. Activation peak for left DLPFC $(x, y, z=-42,9,45)$ lay in the dorsal and posterior part of Brodmann area 9, caudal to BA 46 and rostral to BA 6. TMS coil placement according to the '10-20 method' was used (Kessels et al. 2000; Okamoto et al. 2004). A medium-size 10-20 EEG stretch cap ( ${ }^{\circledR}$.GAMMAcap, http://www.gtec.at) was used to define the 10-20 positions.

Left IPC was reached by placing the coil directly over the P3 electrode position (Kessels et al. 2000; Muri et al. 2002; Vesia et al. 2010). A number of studies (Gerloff et al. 1997; Rossi et al. 2001; Herwig et al. 2003) identified electrode F3 as the closest electrode to target the left DLPFC. We corrected for the relatively posterior location of the left DLPFC activation in previous neuroimaging studies using the present paradigm $(x, y, z=-42,9,45$ in MNI space; Chambon et al. 2013) by targeting a site located $12 \mathrm{~mm}$ posterior to $\mathrm{F} 3$ and $12 \mathrm{~mm}$ anterior to FC3. In Sham stimulation trials, the coil was held over the vertex defined by the $10 / 20$ system, but was rotated through 90 degrees, so that no current was induced in the brain.

(b) Stimulation intensity TMS over most cortical areas other than primary visual and primary motor does not produce reliably reportable or observable effects on individual trials. The optimal methods for selecting stimulation intensity for non-motor areas remain unclear. Since our interest focussed on sense of agency accompanying voluntary actions, we particularly wanted to avoid inducing involuntary movements such as hand twitches, which are known to undermine sense of agency (Haggard et al. 2002; Haggard and Clark 2003). Some studies have linked levels of non-motor stimulation to the resting motor threshold. However, the motor threshold to TMS may not adequately represent the excitability of non-motor areas of the brain 
(Boroojerdi et al. 2002; Robertson et al. 2003; Oliver et al. 2009). In addition, we were concerned that this method would not sufficiently guard against involuntary TMSinduced movements, particularly for stimulations delivered around the time of voluntary action. Therefore, intensity of magnetic stimulation for IPC and DLPFC was defined individually prior to the experiment. Thus, intensity of magnetic stimulation was gradually increased by $5 \%$ (e.g., $40 \%$, then $45 \%$, then $50 \%$, etc.) until the stimulation produced twitches or was reported as uncomfortable by the participant. Once this threshold was reached, intensity was gradually decreased by $1 \%$ (e.g., 49, 48, 47, etc.) up to a point where the twitch was no longer present and/or the participant no longer felt the discomfort. On average, this level was at an intensity of $53 \%$ of the stimulator output for IPC (mean $=52.91, \mathrm{SD}=2.46$ ). This value is broadly consistent with that used in other studies stimulating the same region (Vesia et al. 2006, 2008; Buelte et al. 2008; Prime et al. 2008). Using the same criterion, the intensity of stimulation for DLPFC was rather lower (mean $=46.0$, $\mathrm{SD}=2.44$ ). Intensity of the stimulator output in the Sham condition was the same as that used in IPC condition (mean $=52.91, \mathrm{SD}=2.46$ ). The effect of interindividual variations in stimulation intensity on behavioural performance was tested in a sensitivity analysis (see below, "Results" section).

All stimulation parameters were in accordance with the safety guidelines for magnetic stimulation (Wassermann 1998). After completing the experiment, none of the participants reported any undesirable side effects as a result of the stimulation.

Data analyses

Error rates (ERs) and response times (RTs) were analysed independently using a $2 \times 3$ repeated-measures ANOVA with prime-target compatibility (compatible vs. incompatible) and stimulation site (DLPFC vs. IPC vs. Sham) as within-subjects factors. Control ratings for colour effects were analysed using a $2 \times 3 \times 3$ repeated-measures ANOVA with prime-target compatibility, stimulation site, and action-effect interval $(100,300,500 \mathrm{~ms})$ as withinsubjects factors. Post-hoc Fisher tests were used to identify differences between conditions. Finally, we performed correlation analyses between control ratings and intensity of the stimulation for each TMS location (IPC and DLPFC).

Results

\section{Reaction times}

Participant's responses to arrow target following compatible primes were faster than following incompatible primes [main effect of compatibility: $\quad F(1,11)=33.01$, $p<0.001]$. Neither the main effect of stimulation site $[F(2,22)=0.38, p=0.68]$ nor the interaction between compatibility and stimulation site was significant $[F(2,22)=1.16, p=0.33]$, indicating that TMS applied to either left IPC or DLPFC did not alter the facilitation effect of prime-target compatibility on mean RT that normally occurs under no-TMS (or 'Sham') conditions (see: Wenke et al. 2010; Chambon et al. 2013).

\section{Error rates}

Participants made more errors in incompatible than in compatible trials [main effect of compatibility: $F(1,11)=8.68, p=0.01]$. As for response times, neither the main effect of stimulation site $[F(2,22)=2.78$, $p=0.08]$ nor the interaction between compatibility and simulation site was significant $[F(2,22)=1.59, p=0.22]$.

\section{Control ratings}

Participants experienced higher levels of control over action effects following compatible prime-target associations [main effect of compatibility: $F(1,11)=5.77$, $p=0.03$ ], consistent with previous results (e.g., Chambon et al. 2013). Importantly, this effect of prime-target compatibility on control ratings was modulated by the simulation site $[F(2,22)=3.62, p=0.04]$ (Fig. 2). Thus, posthoc tests showed that TMS applied to left IPC effectively abolished the effect of prime-target compatibility on control ratings $(p=0.86)$, whereas the compatibility effect remained highly significant in the Sham $(p=0.009)$ and

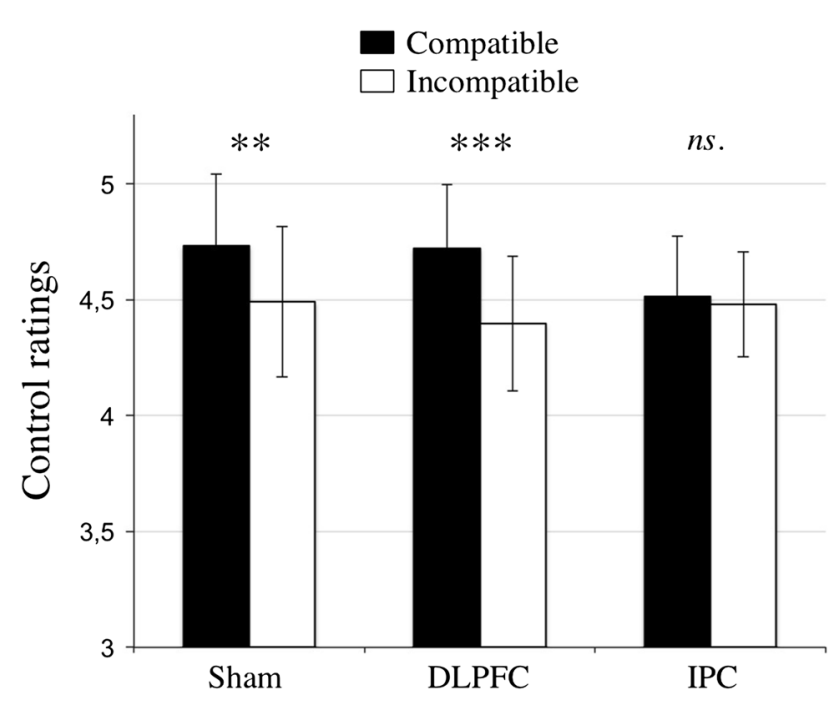

Fig. 2 Mean control ratings on compatible and incompatible trials, for each stimulation site. All error bars indicate SD. $* * p<0.01$; $* * * p<0.001 ;{ }^{\mathrm{ns}} p>0.05$ 
DLPFC $\quad(p=0.0008)$ conditions. Comparing trials between stimulation sites revealed an effect of TMS stimulation on compatible trials only, with lower control ratings in these trials when TMS was applied to IPC compared to DLPFC or Sham (compatible trials: IPC vs. Sham, $p=0.009$; IPC vs. DLPFC, $p=0.01)$, and no other significant differences.

In addition, we found a predicted main effect of action-effect interval $[F(2,22)=4.38, p=0.02]$. Posthoc testing showed that participants experienced stronger sense of agency with 100-ms response-effect intervals than with $500 \mathrm{~ms}(p=0.007)$. Importantly, predictability of the effect did not differently influence sense of agency across compatible and incompatible trials, as there was no significant interaction between action-effect interval and compatibility $[F(2,22)=0.84, p=0.44]$. Finally, neither the interaction between action-effect interval and stimulation site $[F(4,44)=1.63, p=0.18]$ nor the threeway interaction between all the factors $[F(4,44)=0.35$, $p=0.83$ ] was significant. Thus, TMS applied to IPC disrupted the influence of action-selection compatibility on sense of agency, irrespective of post action-selection components, such as the action-effect interval.

\section{Sensitivity analyses}

Stimulation intensity was set for each participant and each site separately. Therefore, we investigated whether the size of the compatibility effect in each condition might simply reflect the variations in TMS intensity. We calculated a (net) compatibility effect by subtracting control ratings in incompatible trials from those obtained in compatible trials for each stimulation site (IPC and DLPFC). We then correlated the compatibility effect and intensity of TMS across participants in each condition. We found positive correlations between stimulation intensity and compatibility effects on control ratings, but these were weak, and far from statistically significant (IPC: $R=0.27, p=0.39$; DLPFC: $R=0.20, p=0.51)$. Thus, IPC stimulation effectively abolished the group mean compatibility effect, irrespective of individual variations in TMS intensity. This combination of results suggests that the presence of IPC stimulation, rather than our method of individual stimulation intensity adjustment, was responsible for the change in control ratings.

The positive correlation between TMS intensity and compatibility effect is also relevant for interpreting the DLPFC data. The null effect of DLPFC stimulation on control ratings might, in principle, be a simple consequence of the lower intensity of stimulation used for DLPFC, compared to IPC. In that case, DLPFC stimulation intensity should be directly related to compatibility effects on control ratings, yet no such effect was found.
Preliminary discussion

Previous studies have shown that prime-target compatibility modulates the sense of agency over the external consequences of movement (Wenke et al. 2010; Chambon and Haggard 2012; Chambon et al. 2013). Incompatible prime-target pairings reduce the sense of agency, an effect that is associated with increased activity in the left IPC (Chambon et al. 2013). The results from the present study confirm the role of left IPC, showing that TMS-induced disruption of activity within this region essentially abolished the compatibility effect. Importantly, TMS had no effect on reaction times. Thus, while TMS stimulation had significant effects on sense of agency, it did not influence task performance. There are several other examples of dissociation between sense of agency and action performance. For example, changing the timing parameters of masking has profound effects on action performance (reversing the positive compatibility effect to produce a 'negative compatibility effect', resulting in increased response latencies), but leaves the priming effects on sense of agency unchanged (Chambon and Haggard 2012; Stenner et al. 2014). Sense of agency and motor control could rely on different components and would recruit different circuits accordingly. Thus, TMS over IPC would not interfere with action selection processing itself, since that would presumably influence performance. Rather, TMS over IPC would interfere with a circuit that monitors action selection signals to construct the experience of control-a possibility we suggested in a previous model (Chambon and Haggard 2012). Finally, there were no significant effects of left DLPFC stimulation. Because the level of stimulation was lower for DLPFC than for IPC, due to participant acceptability, we cannot conclude that DLPFC stimulation is ineffective.

Traditionally, IPC is thought to play a role in late retrospective aspects of sense of agency by monitoring the consistency between predicted and actual sensory consequences of movement (David et al. 2008, for a review). However, the present findings suggest that the IPC may also be involved in earlier prospective aspects of sense of agency. In "Experiment 2" we directly investigated this by varying the timing of TMS over left IPC. Stimulation was either delivered $70 \mathrm{~ms}$ after target onset, TMS delivered $70 \mathrm{~ms}$ after the keypress, or TMS delivered $70 \mathrm{~ms}$ after the presentation of the colour effect (there was also a nostimulation condition). If the IPC is involved in prospective aspects of sense of agency then the effect of stimulation should be most pronounced in the earlier stages of action execution. Owing to the lack of an effect of DLPFC stimulation in "Experiment 1" we focussed only on the IPC. 
Fig. 3 a, b Experimental procedure. a Site of TMS stimulation (left IPC at the coordinate location of Chambon et al. 2013). b Example trials from the two possible combinations of the primeaction compatibility (compatible upper panel; incompatible lower panel). TMS was delivered $70 \mathrm{~ms}$ after either mask/target onset (action selection), or action onset (action execution), or after the presentation of the action-effect (a)
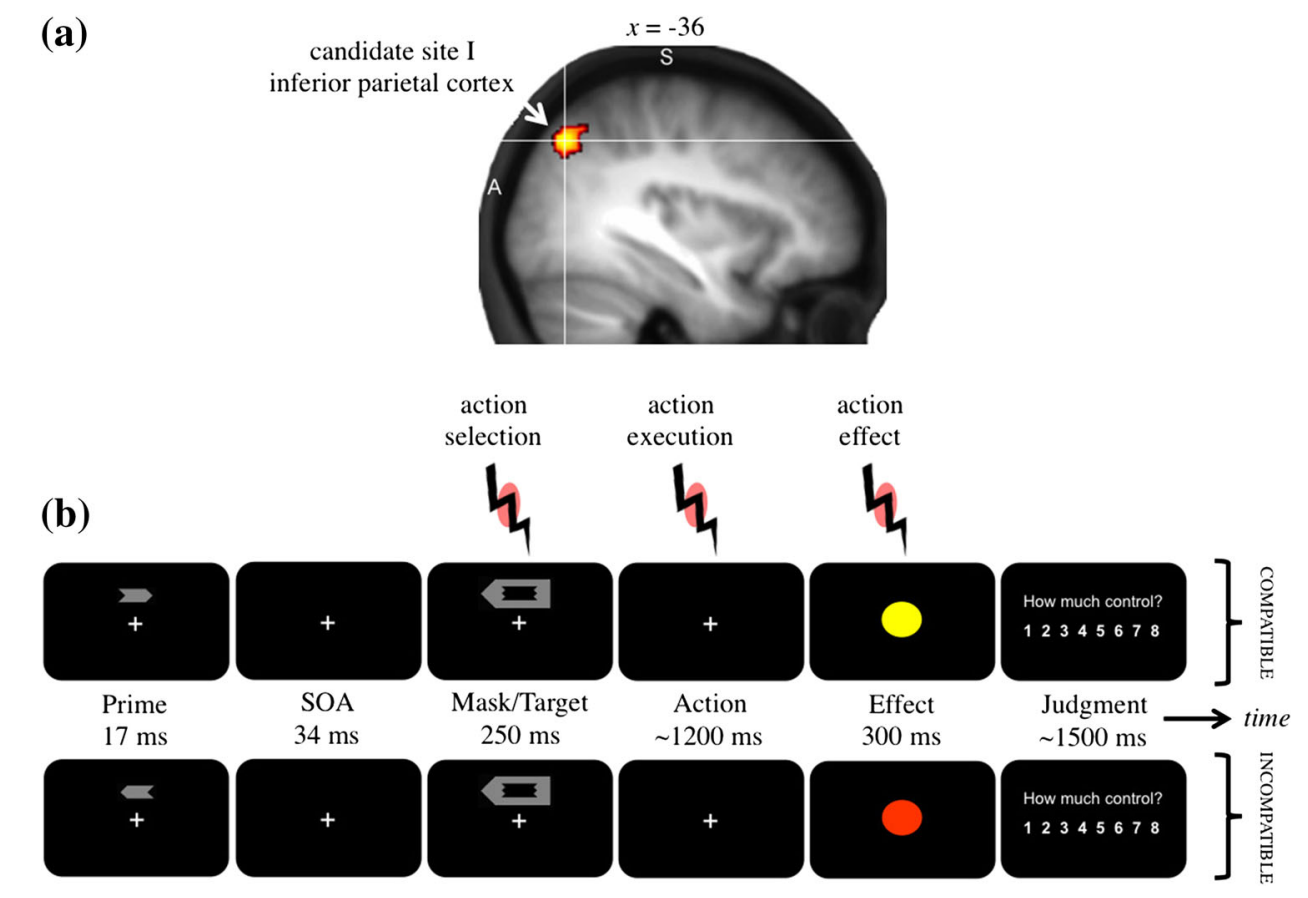

\section{Experiment 2}

Material and Methods

\section{Participants}

Twelve participants (6 females and 6 males aged 21-42 years), different from those tested in "Experiment 1 ", with normal or corrected-to-normal vision, were recruited to participate in the study. They provided written informed consent prior to the experiment and were paid $£ 10$ for their participation. All participants were without any known contra-indications to TMS. The experiment was approved by the UCL Research Ethics Committee and has therefore been performed in accordance with the ethical standards laid down in the 1964 Declaration of Helsinki.

\section{Behavioural task}

The behavioural task (stimuli, timeline, and instructions) was identical to that used in Experiment 1.

\section{TMS methods}

TMS was only applied to the left IPC. The location (electrode P3) was identical to that used in "Experiment 1 ". The intensity of the stimulation was set as before (mean $=53.08, \mathrm{SD}=2.39)$ and did not significantly differ from that used in the first experiment $(p=0.88)$. Only the timing of stimulation changed. Thus, during the trial there was either: no TMS stimulation, TMS delivered $70 \mathrm{~ms}$ after target onset, TMS delivered $70 \mathrm{~ms}$ after the keypress, or TMS delivered $70 \mathrm{~ms}$ after the presentation of the colour effect (Fig. 3). The timing of stimulation was randomised across trials.

Data analyses

Error rates (ERs), response times (RTs), and control ratings were analysed independently using a $2 \times 4$ repeatedmeasures ANOVA with prime-target compatibility (compatible vs. incompatible) and timing of stimulation (NoTMS vs. target vs. keypress vs. effect) as within-subjects factors. Post-hoc Fisher tests were used to identify differences between conditions. Finally, as in "Experiment 1", we performed correlation analyses between control ratings and intensity of the stimulation over IPC.

Results

\section{Reaction times}

The main effect of prime-target compatibility was significant $[F(1,11)=33.15, p<0.001]$. Neither the main effect of the timing of stimulation $[F(3,33)=0.42$, $p=0.73$ ] nor the interaction between compatibility and timing was significant $[F(3,33)=0.15, p=0.92]$.

\section{Error rates}

Participants tended to make more errors in incompatible than in compatible trials [main effect of compatibility: 
$F(1,11)=4.2, p=0.06]$. As for response times, neither the main effect of the timing of stimulation $[F(3,33)=0.14$, $p=0.93]$ nor the interaction between compatibility and timing was significant $[F(3,33)=0.18, p=0.9]$.

\section{Control ratings}

The main effect of prime-target compatibility $[F(1,11)=3.75, p=0.07]$, as well as the main effect of the timing of stimulation $[F(3,33)=0.8, p=0.49]$, did not reach significance. Importantly, however, the effect of compatibility on control was significantly influenced by the timing of stimulation, as shown by the interaction between the two factors $[F(3,33)=4.16, p=0.01]$ (Fig. 4). Thus, TMS applied to IPC shortly after target, or after response onset reversed or largely abolished ( $p=0.3,0.58$ respectively) the effect of compatibility. In contrast, clear effects of prime compatibility on control ratings were found in the No-TMS condition $(p<0.001)$, or when TMS was applied shortly after presentation of the action effect $(p=0.02)$. Comparing trials across the different timings of stimulation revealed an effect of TMS stimulation on incompatible trials only with higher control ratings for post-target TMS than for post-effect TMS ( $p=0.03$ ), and also for post-target TMS compared to no TMS $(p=0.001)$.

\section{Sensitivity analyses}

Correlation analyses between the compatibility effect and the intensity of the stimulator output for each timing of stimulation did not reveal any significant associations,

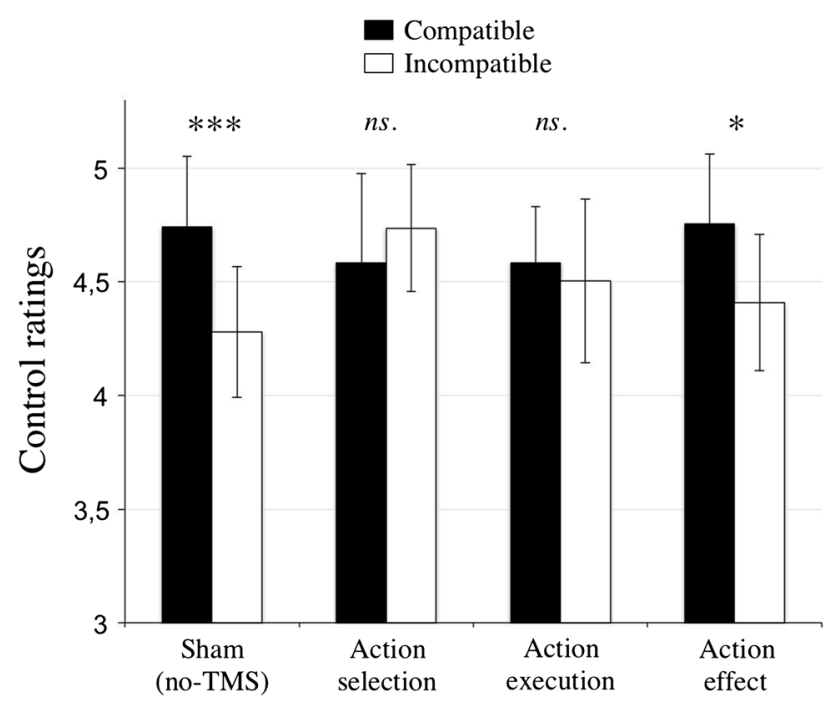

Fig. 4 Mean control ratings for compatible and incompatible trials, for each timing of stimulation, and for the No-TMS condition. All error bars indicate SD. $* p<0.05 ; * * * p<0.001 ;{ }^{n} p>0.05$ whether TMS was delivered after target onset $(R=0.11$, $p=0.71)$, action onset $(R=0.25, p=0.42)$, or actioneffect onset $(R=-0.31, p=0.32)$.

\section{Preliminary discussion}

The results from "Experiment 1" suggested that left IPC may be involved in early prospective aspects of sense of agency. In "Experiment 2" we directly contrasted prospective and retrospective contributions to sense of agency by varying the timing of TMS over IPC. It was found that the compatibility effect was abolished following stimulation delivered $70 \mathrm{~ms}$ after target onset and also $70 \mathrm{~ms}$ after the keypress. However, the compatibility effect re-emerged if stimulation was delivered $70 \mathrm{~ms}$ after the presentation of the colour effect. Crucially, TMS again had no effect on RTs, ruling out interpretations based on disruption of action execution. This pattern of results provides compelling evidence that left IPC is causally involved in computing sense of agency, that these computations involve prospective, premotor information, and that these computations occur around the time of action selection and execution. The prospective contribution of left IPC to sense of agency can thus be distinguished from other functions such as action outcome monitoring. Interestingly, Farrer et al. (2008) demonstrated a role of IPC in action outcome monitoring, but found a bilateral IPC activation, which was slightly more ventral than the IPC stimulation site used here. In Farrer et al.'s study, IPC activation varied with mismatch between predicted and actual sensory consequences of an action, while IPC activation in our previous fMRI study was elicited by a mismatch between a primeinduced intention and response to a target (Chambon et al. 2013). The different localisation found in Farrer et al.'s study and ours could thus reflect a subdivision within the inferior parietal cortex, with more dorsal IPC being involved in detecting mismatch between intention and action, while more ventral IPC would be involved in comparing predicted and actual consequences of an action.

\section{General discussion}

Previous studies have shown that the fluency or dysfluency of action selection influences the sense of agency over an external sensory event (Wenke et al. 2010; Chambon and Haggard 2012; Haggard and Chambon 2012; Chambon et al. 2013). Furthermore, recent fMRI data suggest that this early prospective contribution to sense of agency is supported by a network involving left DLPFC and left IPC (Chambon et al. 2013). We directly tested this hypothesis, using single-pulse TMS to selectively disrupt activity in DLPFC or IPC. In a first experiment, we confirmed the 
importance of left IPC by showing that TMS here abolished the priming effect on judgments of control. TMS over left DLPFC had no significant effect on task performance. In a second experiment, we showed that this contribution of left IPC is limited to early preparation and execution phases of movement.

In our study, the inferior parietal cortex was targeted by the P3 electrode position, as in previous studies (Kessels et al. 2000; Muri et al. 2002; Vesia et al. 2010). One recent study argued that this method carries a much greater type II error than targeting based on fMRI localisers (Sack et al. 2009), at least for studies of numerical cognition. However, the loss of power associated with scalp-based neurotargeting must depend on the localisation in the brain of the cognitive function under study. When there are large individual differences in the brain area where a function is localised (e.g., Nieto-Castañón and Fedorenko 2012), coil placement based on individual fMRI is clearly preferable. However, scalp-based coil placement may be relatively successful where individual differences in functional localisation are small, and where the relation between scalp location and underlying brain area is known and stable. Interestingly, we found a significant effect of TMS on compatibility-related sense of agency in "Experiment 1", despite the relatively small number of participants and the allegedly low power of the coil placement technique. Moreover, we then replicated this effect in a new group of participants in "Experiment 2".

The results of the present study confirm and extend the findings from a recent fMRI study (Chambon et al. 2013). By abolishing the compatibility effect with TMS over IPC we provide causal evidence of its role in prospective aspects of sense of agency. Furthermore, by exploiting the temporal specificity of single-pulse TMS we were able to pinpoint the precise timing of IPC involvement. The results show, for the first time, that the contribution of IPC to the sense of agency includes early, prospective components related to action selection and action programming. The contribution of IPC to prospective aspects of agency did not depend on processing at the time of action outcomes, because TMS at the time of action outcomes did not influence compatibility effects on control ratings.

Previously it has been suggested that the IPC (i.e., the angular gyrus) is involved in the retrospective construction of sense of agency by monitoring the consistency between predicted and actual sensory consequences of movements (e.g., Farrer et al. 2008). When these predictions are violated sense of agency is reduced, and IPC activity is increased. Our results do not disagree with this view of IPC function, and are indeed independent of it. Rather, we set out to investigate whether IPC might play an additional role, at the much earlier time-point of action selection. More specifically, our results show that, by monitoring the consistency between action plans and required actions, the IPC is involved in the prospective construction of sense of agency. As suggested above, these two putative functions for IPC may reflect a functional subdivision within the inferior parietal cortex, with more ventral IPC being involved in retrospectively monitoring the link between the action and the consequences of this action (Farrer et al. 2008), while more dorsal IPC would be involved in monitoring the link between intention and action, prior to action execution and independent of action consequences (Chambon et al. 2013).

The prospective and retrospective mechanisms have some general features in common. Both involve monitoring action-related signals or 'cues' as they become available, and comparing them with other relevant information for consistency. We suggest that monitoring and checking is a very general function of the IPC during instrumental action. Previous research has highlighted the contribution of various cues to sense of agency, in particular sensorimotor prediction and re-afferent sensory feedback (Moore et al. 2009). The present results suggest that information generated during the process of action selection is also monitored and checked in IPC. For example, initial action intentions, such as those caused by subliminal primes, could be checked for compatibility with the action subsequently performed. These action selection cues may provide an important 'online' marker of control as the action is unfolding. Not only would this provide an estimate of agency without the need to wait until sensory feedback becomes available, but as Chambon et al. (2013) have suggested, it may also protect against aberrant experiences of agency. For example, the sense of agency in patients with schizophrenia is characterised by excessive reliance on re-afferent sensory information generated by their actions (Voss et al. 2010). Prospective signals, if available, may provide an important counterweight.

Moore and Fletcher (2012) have recently suggested that the normal sense of agency involves the optimal integration of various sources of information. The present results suggest a candidate for the neural implementation of this cue integration. As stated above, the IPC appears to play a very general role in monitoring the consistency between various action-related signals. This would make the IPC ideally suited to the task of implementing cue integration. Our result suggests that action selection fluency may be one such cue.

Chambon et al. (2013) showed that activation of IPC was negatively related to sense of control on incompatible prime-target trials, but unrelated to sense of control on compatible prime-target trials. This is consistent with previous fMRI data suggesting that IPC codes for lack of agency, while its activation in conditions of positive agency remains at baseline levels. That is, agency is a 
default backdrop to normal mental life rather than a distinctive phenomenal experience (Frith et al. 2000; Haggard 2005; Chambon and Haggard 2013). This view predicts that disruption of IPC might attenuate the reduction in sense of agency on incompatible trials only, where prime and target conflict produces a subjective loss of control accordingly. However, inspection of the results from "Experiment 1" suggests that stimulation of IPC also reduced sense of agency on compatible trials. In "Experiment 2", stimulation of IPC again reduced sense of agency on compatible trials, though an increased sense of agency on incompatible trials was now also observed. Including a neutral priming condition in future research would allow a clearer separation between the facilitation of agency by compatible primes and the impairment of agency by incompatible primes. The present study suggests that IPC is involved in the influence of prime compatibility on sense of control, but we cannot definitively say whether IPC involvement reflects a benefit of fluent action selection, a cost of dysfluent action selection, or both.

This study has several limitations. First, stimulation was delivered $70 \mathrm{~ms}$ similarly after arrow presentation, keypress, and colour presentation, to keep the timing of the TMS pulse constant across prospective (response and target onsets) and retrospective (effect onset) conditions, and to allow for strict comparisons across these conditions. However, we cannot exclude the possibility that, whether this stimulation timing was appropriate to alter prospective coding of agency, it might have missed any retrospective component. Indeed, retrospective coding of agency may occur later, as suggested by the temporal course of a wellestablished index of retrospective matching process, i.e., the mismatch negativity (150-250 ms post-stimulus; Garrido et al. 2009). Note however that the disruptive effect of TMS stimulation is thought to last for around 100-200 ms (see Thut et al. 2003, for a review); hence, the temporal window of disruption used here should have hindered any process occurring up to $170-270 \mathrm{~ms}$ after stimulus, response, or effect onset.

Second, although we found compelling evidence in support of the role of IPC, our results showed no statistically reliable effect following stimulation of DLPFC. One possible explanation for this is the high interindividual variability in the exact extent of DLPFC's cytoarchitectonic and functional boundaries (Rajkowska and GoldmanRakic 1995a, b; Sanches et al. 2009. Combined with our scalp-based coil placement, this could potentially increase type II error (Sack et al. 2009). A second factor could be the lower stimulation intensity used for DLPFC compared with IPC, although our sensitivity analyses did not find direct evidence for this. The intensity of magnetic stimulation was determined for each individual by progressively increasing the intensity of the stimulator output up to a point where muscle twitches or significant discomfort occurred. Higher intensity stimulation might potentially have caused a significant increase in compatibility effect following DLPFC stimulation, but such a study might exceed the limits of acceptability.

Third, we used explicit, rather than implicit, judgments of agency. Implicit judgements can also be affected by subliminal action priming (Stenner et al. 2014). Indeed, a disadvantage of explicit judgements is the risk of confounds with other aspects of action experience, such as task performance. We however believe such confound is unlikely to occur here, as prospective sense of agency and performance monitoring have been shown to clearly dissociate (e.g., Chambon and Haggard 2012; Chambon et al. 2013; Sidarus et al. 2013; Damen et al. 2014). Explicit judgement has the merit that one clearly measures sense of agency, as consciously experienced by the subject, while implicit judgements can sometimes be criticised on the grounds that they do not unambiguously index the conscious experience of control (Moore and Sukhvinder 2012).

In summary, we provide, for the first time, direct evidence for the role of IPC in the prospective sense of agency. We found that TMS over IPC abolished the compatibility effect. A second experiment replicated these results, and showed that they were temporally restricted, occurring only at early stages of action preparation and execution. These results show that IPC is involved in construction of sense of agency much earlier than has been previously thought, and in advance of action outcomes. Crucially, our results point to an additional, prospective contribution of IPC to sense of agency, which is independent from its well-established role in matching predicted and actual consequences of action. We have suggested that this would be consistent with a more general role for the IPC in integrating and monitoring multiple action-related signals, including premotor signals.

Acknowledgments JWM and PH were supported by EU project VERE. VC was supported by a postdoctoral fellowship of the Région Ile-de-France (Paris), ANR-10-LABX-0087 IEC and ANR-10-IDEX0001-02 PSL*. PH was supported by a Leverhulme Trust Major Research Fellowship and an ESRC Professorial Fellowship (Grant No. RES-062-23-2183), and ERC Advanced Grant HUMVOL.

\section{References}

Amassian VE, Cracco RQ, Maccabee PJ, Cracco JB, Rudell AP, Eberle L (1998) Transcranial magnetic stimulation in study of the visual pathways. J Clin Neurophysiol 15:288-403

Blakemore SJ, Wolpert D, Frith C (1998) Central cancellation of selfproduced tickle sensation. Nat Neurosci 1:635-640

Boroojerdi B, Meister IG, Foltys H, Sparing R, Cohen LG, Topper R (2002) Visual and motor cortex excitability: a transcranial magnetic stimulation study. Clin Neurophysiol 113:1501-1504 
Buelte D, Meister IG, Staedtgen M, Dambeck N, Sparing R, Grefkes C, Boroojerdi B (2008) The role of the anterior intraparietal sulcus in crossmodal processing of object features in humans: an rTMS study. Brain Res 1217:110-118

Chambon V, Haggard P (2012) Sense of control depends on fluency of action selection, not motor performance. Cognition 125:441-451

Chambon V, Haggard P (2013) Premotor or ideomotor: how does the experience of action come about? In: Prinz W, Beisert M, Herwig A (eds) Action science: foundations of an emerging discipline. MIT Press, Cambridge

Chambon V, Wenke D, Fleming SM, Prinz W, Haggard P (2013) An online neural substrate for sense of agency. Cereb Cortex 23:1031-1037

Damen TG, van Baaren RB, Djiksterhuis A (2014) You should read this! Perceiving and acting upon action primes influences one's sense of agency. J Exp Soc Psychol 50:21-26

David N, Cohen MX, Newen A, Bewernick BH, Shah NJ, Fink GR, Vogeley K (2007) The extrastriate cortex distinguishes between the consequences of one's own and others' behavior. NeuroImage 36:1004-1014

David N, Newen A, Vogeley K (2008) The "sense of agency" and its underlying cognitive and neural mechanisms. Conscious Cogn 17:523-534

Dehaene S, Artiges E, Naccache L, Martelli C, Viard A, Schürhoff F, Recasens C, Martinot ML, Leboyer M, Martinot JL (2003) Conscious and subliminal conflicts in normal subjects and patients with schizophrenia: the role of the anterior cingulate. Proc Natl Acad Sci 100:13722-13727

Farrer C, Franck N, Georgieff N, Frith CD, Decety J, Jeannerod M (2003) Modulating the experience of agency: a positron emission tomography study. NeuroImage 18:324-333

Farrer C, Frey SH, Van Horn JD, Tunik E, Turk D, Inati S, Grafton ST (2008) The angular gyrus computes action awareness representations. Cereb Cortex 18:254-261

Fourneret P, Jeannerod M (1998) Limited conscious monitoring of motor performance in normal subjects. Neuropsychologia 36:1133-1140

Franck N, Farrer C, Georgieff N, Marie-Cardine M, Dalery J, d'Amato T, Jeannerod M (2001) Defective recognition of one's own actions in patients with schizophrenia. Am J Psychiatry 158:454-459

Frith CD, Friston K, Liddle PF, Frackowiak RSJ (1991) Willed action and the prefrontal cortex in man: a study with PET. Proc R Soc Lond B Bio Sci 244:241-246

Frith CD, Blakemore SJ, Wolpert DM (2000) Abnormalities in the awareness and control of action. Philos Trans R Soc Lond B Biol Sci 355:1771-1788

Garrido MI, Kilner JM, Stephan KE, Friston KJ (2009) The mismatch negativity: a review of underlying mechanisms. Clin Neurophysiol 120:453-463

Gentsch A, Schütz-Bosbach S (2011) I did it: unconscious expectation of sensory consequences modulates the experience of self-agency and its functional signature. J Cogn Neurosci 23:3817-3828

Gerloff C, Corwell B, Chen R, Hallett M, Cohen LG (1997) Stimulation over the human supplementary motor area interferes with the organization of future elements in complex motor sequences. Brain 120:1587-1602

Haggard P (2005) Conscious intention and motor cognition. Trends Cogn Sci 9:290-295

Haggard P, Chambon V (2012) Sense of agency. Curr Biol 22:390-392

Haggard P, Clark S (2003) Intentional action: conscious experience and neural prediction. Conscious Cogn 12:695-707

Haggard P, Tsakiris M (2009) The experience of agency: feelings, judgements, and responsibility. Curr Dir Psychol Sci 18:242-246
Haggard P, Clark S, Kalogeras J (2002) Voluntary action and conscious awareness. Nat Neurosci 5:382-385

Herwig U, Satrapi P, Schonfeldt-Lecuona C (2003) Using the international 10-20 EEG system for positioning of transcranial magnetic stimulation. Brain Topogr 16:95-99

Kamitani Y, Shimojo S (1999) Manifestation of scotomas created by transcranial magnetic stimulation of human visual cortex. Nat Neurosci 2:767-771

Kammer T, Nusseck HG (1998) Are recognition deficits following occipital lobe TMS explained by raised detection threshold? Neuropsychologia 36:1161-1166

Keel JC, Smith MJ, Wassermann EM (2001) A safety screening questionnaire for transcranial magnetic stimulation. Clin Neurophysiol 112:720

Kessels RP, d'Alfonso AA, Postma A, de Haan EH (2000) Spatial working memory performance after high-frequency repetitive transcranial magnetic stimulation of the left and right posterior parietal cortex in humans. Neurosci Lett 287:68-70

Leube DT, Knoblich G, Erb M, Grodd W, Bartels M, Kircher TT (2003) The neural correlates of perceiving one's own movements. NeuroImage 20:2084-2090

Lingnau A, Vorberg D (2005) The time course of response inhibition in masked priming. Percept Psychophys 67:545-557

Linser K, Goschke T (2007) Unconscious modulation of the conscious experience of control. Cognition 104:459-475

MacDonald PA, Paus T (2003) The role of parietal cortex in awareness of self-generated movements: a transcranial magnetic stimulation study. Cereb Cortex 13:962-967

Martikainen MH, Kaneko K, Hari R (2005) Suppressed responses to self-triggered sounds in the human auditory cortex. Cereb Cortex 15:299-302

Metcalfe J, Greene MJ (2007) Metacognition of agency. J Exp Psychol Gen 136:184-199

Moore JW, Fletcher PC (2012) Sense of agency: a review of cue integration approaches. Conscious Cogn 21:59-68

Moore J, Haggard P (2008) Awareness of action: inference and prediction. Conscious Cogn 17:136-144

Moore JW, Sukhvinder OS (2012) Intentional binding and the sense of agency: a review. Conscious Cogn 21:546-561

Moore JW, Wegner DM, Haggard P (2009) Modulating the sense of agency with external cues. Conscious Cogn 18:1056-1064

Muri RM, Buhler R, Heinemann D, Mosimann UP, Felblinger J, Schlaepfer TE, Hess CW (2002) Hemispheric asymmetry in visuospatial attention assessed with transcranial magnetic stimulation. Exp Brain Res 143:426-430

Nieto-Castañón A, Fedorenko E (2012) Subject-specific functional localizers increase sensitivity and functional resolution of multisubject analyses. Neuroimage 15:1646-1669

Okamoto M, Dan H, Sakamoto K, Takeo K, Shimizu K, Kohno S, Oda I, Isobe S, Suzuki T, Kohyama K, Dan I (2004) Threedimensional probabilistic anatomical cranio-cerebral correlation via the international 10-20 system oriented for transcranial functional brain mapping. Neuroimage 21:99-111

Oliver R, Bjoertomt O, Driver J, Greenwood R, Rothwell J (2009) Novel "hunting" method using transcranial magnetic stimulation over parietal cortex disrupts visuospatial sensitivity in relation to motor thresholds. Neuropsychologia 47:3152-3161

Prime SL, Vesia M, Crawford JD (2008) Transcranial magnetic stimulation over posterior parietal cortex disrupts transsaccadic memory of multiple objects. J Neurosci 28:6938-6949

Rajkowska G, Goldman-Rakic PS (1995a) Cytoarchitectonic definition of prefrontal areas in the normal human cortex: I. Remapping of areas 9 and 46 using quantitative criteria. Cereb Cortex 5:307-322

Rajkowska G, Goldman-Rakic PS (1995b) Cytoarchitectonic definition of prefrontal areas in the normal human cortex: II. 
Variability in locations of areas 9 and 46 and relationship to the Talairach Coordinate System. Cereb Cortex 5:323-337

Robertson EM, Theoret H, Pascual-Leone A (2003) Studies in cognition: the problems solved and created by transcranial magnetic stimulation. J Cogn Neurosci 15:948-960

Rossi S, Cappa SF, Babiloni C, Pasqualetti P, Miniussi C, Carducci F, Babiloni F, Rossini PM (2001) Prefrontal cortex in long-term memory: an "interference" approach using magnetic stimulation. Nat Neurosci 4:948-952

Sack AT, Cohen Kadosh R, Schuhmann T, Moerel M, Walsh V, Goebel R (2009) Optimizing functional accuracy of TMS in cognitive studies: a comparison of methods. J Cogn Neurosci 21:201-221

Sanches M, Caetano S, Nicoletti M, Monkul ES, Chen HH, Hatch JP, Yeh PH, Mullis RL, Keshavan MS, Rajowska G, Soares JC (2009) An MRI-based approach for the measurement of the dorsolateral prefrontal cortex in humans. Psychiatry Res 173:150-154

Sanger TD, Garg RR, Chen R (2001) Interactions between two different inhibitory systems in the human cortex. J Physiol 530:307-317

Sato A (2009) Both motor prediction and conceptual congruency between preview and action-effect contribute to explicit judgement of agency. Cognition 110:74-83

Sidarus N, Chambon V, Haggard P (2013) Priming of actions increases sense of control over unexpected outcomes. Conscious Cogn 22:1403-1411

Stenner MP, Bauer M, Sidarus N, Heinze HJ, Haggard P, Dolan RJ (2014) Subliminal action priming modulates the perceived intensity of sensory action consequences. Cognition 130:227-235

Synofzik M, Thier P, Lindner A (2006) Internalizing agency of selfaction: perception of one's own hand movements depends on an adaptable prediction about the sensory action outcome. J Neurophysiol 96:1592-1601
Synofzik M, Vosgerau G, Newen A (2008) Beyond the comparator model: a multifactorial two-step account of agency. Conscious Cogn 17:219-239

Thut G, Northoff G, Ives JR, Kamitani Y, Pfennig A, Kampmann F, Schomer DL, Pascual-Leone A (2003) Effects of single-pulse transcranial magnetic stimulation (TMS) on functional brain activity: a combined event-related TMS and evoked potential study. Clin Neurophysiol 114:2071-2080

Vesia M, Monteon JA, Sergio LE, Crawford JD (2006) Hemispheric asymmetry in memory-guided pointing during single-pulse transcranial magnetic stimulation of human parietal cortex. J Neurophysiol 96:3016-3027

Vesia M, Yan X, Henriques DY, Sergio LE, Crawford JD (2008) Transcranial magnetic stimulation over human dorsal-lateral posterior parietal cortex disrupts integration of hand position signals into the reach plan. J Neurophysiol 100:2005-2014

Vesia M, Prime SL, Yan X, Sergio LE, Crawford JD (2010) Specificity of human parietal saccade and reach regions during transcranial magnetic stimulation. J Neurosci 30:13053-13065

Vorberg D, Mattler U, Heinecke A, Schmidt T, Schwarzbach J (2003) Different time courses for visual perception and action priming. Proc Natl Acad Sci 100:6275-6280

Voss M, Moore J, Hauser M, Gallinat J, Heinz A, Haggard P (2010) Altered awareness of action in schizophrenia: a specific deficit in predicting action consequences. Brain 133:3104-3112

Wassermann EM (1998) Risk and safety of repetitive transcranial magnetic stimulation: report and suggested guidelines from the international workshop on the safety of repetitive transcranial magnetic stimulation. Electroencephalogr Clin Neurophysiol 108:1-16

Wenke D, Fleming SM, Haggard P (2010) Subliminal priming of action influences sense of control over effects of action. Cognition 115:26-38 